\title{
HSC/03/01
}

\section{Modeling electricity prices: Jump diffusion and regime switching}

\author{
Rafał Weron* \\ Michael Bierbrauer** \\ Stefan Trück**
}

* Hugo Steinhaus Center, Wrocław University of

Technology, Poland

** Chair of Econometrics and Statistics, University of

Karlsruhe, Germany

Hugo Steinhaus Center

Wrocław University of Technology

Wyb. Wyspiańskiego 27, 50-370 Wrocław, Poland

http://www.im.pwr.wroc.pl/ hugo/ 


\title{
Modeling electricity prices: jump diffusion and regime switching
}

\author{
R. Weron ${ }^{\mathrm{a}, 1}$, M. Bierbrauer ${ }^{\mathrm{b}}$, S. Trück ${ }^{\mathrm{b}}$ \\ ${ }^{a}$ Hugo Steinhaus Center for Stochastic Methods, Wrockaw University of \\ Technology, 50-370 Wroctaw, Poland \\ ${ }^{\mathrm{b}}$ Chair of Econometrics and Statistics, University of Karlsruhe, Karlsruhe, 76128, \\ Germany
}

\begin{abstract}
In this paper we address the issue of modeling spot electricity prices. After summarizing the stylized facts about spot electricity prices, we review a number of models proposed in the literature. Afterwards we fit a jump diffusion and a regime switching model to spot prices from the Nordic power exchange and discuss the pros and cons of each one.
\end{abstract}

Key words: electricity price, jump diffusion, regime switching, seasonality PACS: 05.45.Tp, 89.30.-g, 89.65.Gh

\section{Introduction}

The last decade has witnessed radical changes in the structure of the power markets world-wide. Electricity has transformed from a primarily technical business, to one in which the product is treated in much the same way as any other commodity, with trading and risk management as key tools to run a successful business [1-4]. However, for the modeling of electricity prices and the valuation of electricity derivatives we cannot simply rely on models developed for financial or other commodity markets. Electricity is non-storable (at least not economically), which causes demand and supply to be balanced on a knife-edge. Relatively small changes in load or generation can cause large changes in price and all in a matter of hours, if not minutes. In this respect there is no other market like it.

$\overline{1}$ Corresponding author. E-mail address: rweron@im.pwr.wroc.pl.

Preprint submitted to Physica A

Forthcoming in 2004 
All this calls for adequate models of price dynamics capturing the main characteristics of electricity prices. In this paper we address the issue of modeling spot prices, because spot prices are one of the key factors in strategic planning and decision support systems of a majority of market players. After summarizing the stylized facts about spot electricity prices, in Section 3 we review the jump diffusion and regime switching models proposed in the literature to date. In Section 4 we fit two such models to spot prices from the Nordic power exchange and discuss the pros and cons of each one.

\section{Stylized facts about spot electricity prices}

\subsection{The spot market}

The spot electricity market is actually a day-ahead market. A classical spot market would not be possible, since the system operator needs advanced notice to verify that the schedule is feasible and lies within transmission constraints. The spot is normally an hourly contract with physical delivery and is not traded on a continuous basis, but rather in the form of a conducted once per day auction. It is the underlying of most electricity derivatives.

Several countries have deregulated their power markets in the last decade. Yet, typically there are only one or two years of "stationary" data available due to the regulatory changes which are constantly taking place. In our analysis, therefore, we use spot prices from the Nordic power exchange - Nord Pool, which is one of the oldest and generally regarded as the most mature and "stable" power market in the world. Nord Pool offers two types of standardized contracts - physically settled spot contracts and financially settled futures, forward, option and other specialized contracts. Every day is divided into 24 hourly spot contracts. Before noon, the previous day, all participants send in their bids for each hour. The system price is calculated as the equilibrium point for the aggregated supply and demand curves and for each of the 24 hours. It is a theoretical price in the sense that it assumes that no congestions will occur and is the same in the whole Nordic area [5].

\subsection{Seasonality}

It is well known that electricity demand exhibits seasonal fluctuations [1-4]. They mostly arise due to changing climate conditions, like temperature and the number of daylight hours. In some countries also the supply side shows seasonal variations in output. Hydro units, for example, are heavily depen- 
dent on precipitation and snow melting, which varies from season to season. These seasonal fluctuations in demand and supply translate into the seasonal behavior of spot electricity prices. In the top panel of Fig. 1, we have plotted the Nord Pool market daily average system prices since January 1, 1997 until April 25, 2000. Superimposed on the plot is the annual cycle obtained through a wavelet decomposition technique, i.e. a technique consisting of removing several layers of noise and leaving out only the large scale wavelets [6-8]. As it turns out, in this period the annual cycle can be quite well approximated by a sinusoid with a linear trend, see Section 4. This is in line with the approach of Pilipovic [1], who suggests fitting a proper sinusoidal function (eg. a sum of two cosine functions with distinct periods) to spot prices. However, such an approach would not be suitable for some power markets like the German one, where no clear annual seasonality is present and the spot prices behave similarly throughout the year with peaks occurring sometimes in the winter (December 2001 and December-January 2002) and, surprisingly, sometimes in the summer (July 2002 and July-August 2003). Another method of modeling seasonality consists of fitting a piecewise constant function of a one year period, where for each month one tries to determine an average value out of the whole analyzed time series $[9,10]$. Although flexible, this method lacks smoothness, which may have a negative impact on statistical inference of the deseasonalized price process.

\subsection{Mean reversion}

Energy spot prices are in general regarded to be mean reverting [11]. Among other financial time series spot electricity prices are perhaps the best example of anti-persistent data, see $[8,12-14]$ where the R/S analysis, Detrended Fluctuation Analysis (DFA), Average Wavelet Coefficient (AWC) and periodogram regression methods were used to verify this claim. For time intervals ranging from a day to almost four years the Hurst exponent $H$ was found to be significantly lower than 0.5 , indicating mean reversion. For time intervals of less than 24 hours, however, $H$ is above 0.5 , suggesting persistence on the intra-daily level [14].

\subsection{Jumps}

In addition to mean reversion and strong seasonality on the annual, weekly and daily level, spot electricity prices exhibit infrequent, but large jumps. The spot price can increase tenfold during a single hour. Jumps in the spot prices are an effect of extreme load fluctuations, caused by severe weather conditions often in combination with generation outages or transmission failures. These 

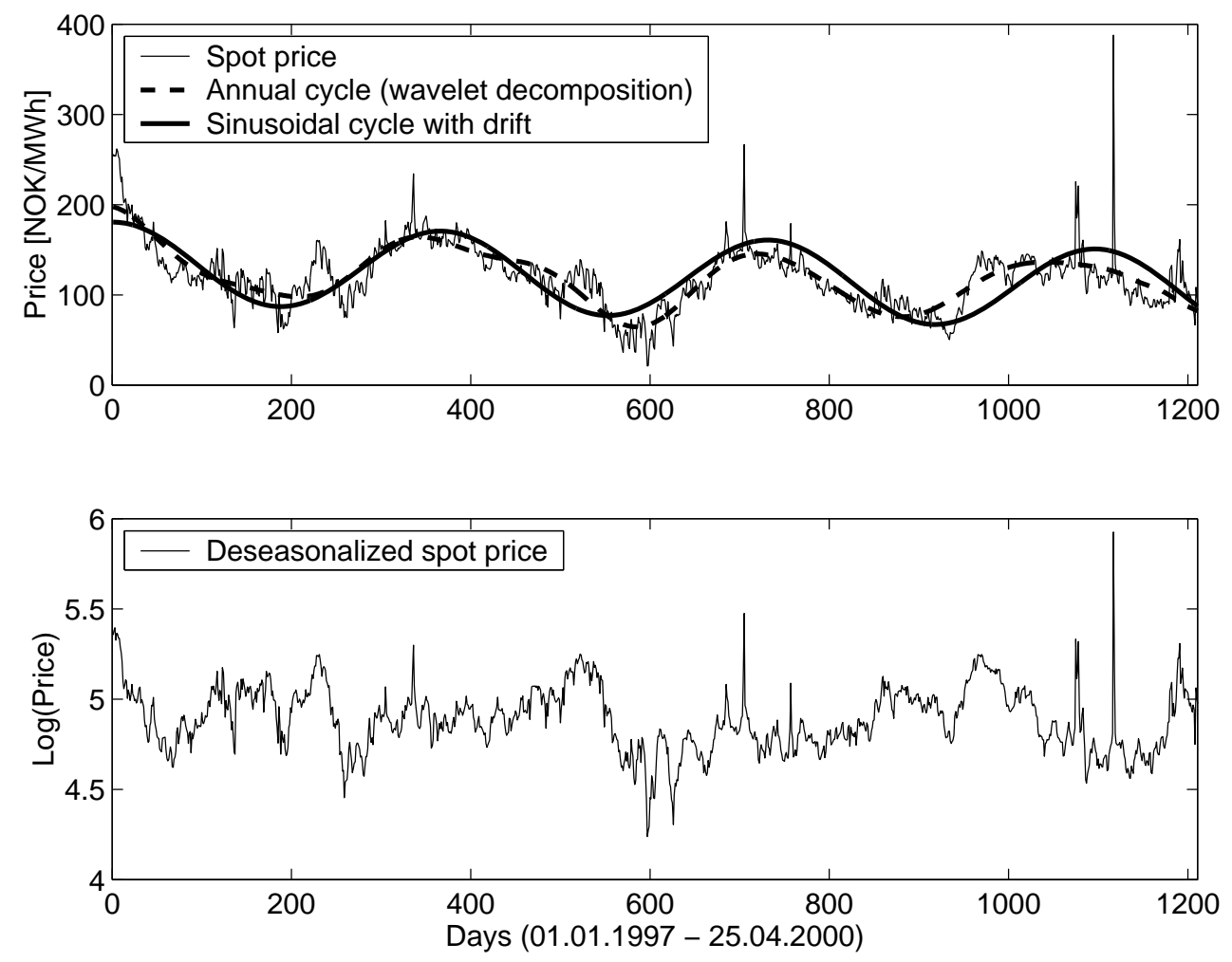

Fig. 1. Top panel: Nord Pool market daily average system prices since January 1, 1997 until April 25, 2000. Superimposed on the plot is the annual cycle obtained through a wavelet decomposition technique and its approximation by a sinusoid with a linear trend. Observe that the sinusoid approximates the annual cycle quite well justifying its use in the analysis. Bottom panel: The deseasonalized log-spot price $d_{t}$ for the same time period.

spikes are normally quite short-lived, and as soon as the weather phenomenon or outage is over, prices fall back to a normal level $[2,8]$.

\section{Spot price modeling}

Having discussed the properties of spot electricity prices we can review the standard modeling approaches and their possible power market modifications. The starting point is the diffusion-type stochastic differential equation (SDE) which, since the pioneering work of Bachelier [15] in 1900, is the standard approach to modeling price processes of stochastic nature. In its most general form the diffusion-type SDE can be written as:

$$
d X_{t}=\mu(X, t) d t+\sigma(X, t) d B_{t}
$$


where $\mu(X, t)$ is the drift, $\sigma(X, t)$ is the volatility (scaling factor) and $d B_{t}$ are the increments of standard Brownian motion. Some phenomena are modeled by a set of related SDE's, leading to so called multi-factor models. On the other hand, both $\mu$ and $\sigma$ can be defined by SDE's of their own, leading to stochastic drift and/or volatility models. Probably the best known member of this family of processes is geometric Brownian motion, with $\mu(X, t)=\mu X$ and $\sigma(X, t)=\sigma X$, introduced to finance by Samuelson [16].

Mean reversion is typically modeled by having a drift term that is negative if the spot price is higher than the mean reversion level and positive if it is lower. To make things easy we start with a very simple mean reverting model originally proposed for specifying interest rate dynamics. The Vasicek [17] model, also referred to as an arithmetic Ornstein-Uhlenbeck process, is described by the following SDE:

$$
d X_{t}=\left(\alpha-\beta X_{t}\right) d t+\sigma d B_{t}=\beta\left(L-X_{t}\right) d t+\sigma d B_{t}
$$

This is a one-factor model that reverts to the mean $L=\frac{\alpha}{\beta}$ with $\beta$ being the magnitude of the speed of adjustment. The second term is responsible for the volatility of the process. The conditional distribution of $X$ at time $t$ is normal with mean $E\left[X_{t}\right]=\frac{\alpha}{\beta}+\left(X_{0}-\frac{\alpha}{\beta}\right) e^{-\beta t}$ and variance $\operatorname{Var}\left[X_{t}\right]=\frac{\sigma^{2}}{2 \beta}\left(1-e^{-2 \beta t}\right)$. These relations imply that $E\left[X_{t}\right] \rightarrow L=\frac{\alpha}{\beta}$ as $t \rightarrow \infty$. Starting at different points the Vasicek model trajectories tend to reverse to the long run mean and stabilize in the corridor defined by the standard deviation of the process. The equilibrium level $L$ can be also made time dependent to reflect the fact that electricity prices tend to revert to different levels over the year.

The "jumpy" character of electricity prices calls for spot price modeling which is not continuous. One approach is to introduce to eqn. (2) a jump component $J_{t} d q_{t}[3,18]$, where $J_{t}$ is a random jump size, eg. a lognormal random variable $\log J_{t} \sim N\left(\mu, \rho^{2}\right)$, and $q_{t}$ is a Poisson random variable with intensity $\kappa$. Eydeland and Geman [4] propose a similar model, where - to account for the fact that jumps tend to be more severe during high price periods - the jump part is given by $J_{t} X_{t} d q_{t}$. In these models the price after a spike is forced back to its normal level by the mean reversion mechanism. However, this may be not fast enough. Geman and Roncoroni [19] suggest using mean reversion coupled with downward jumps. Alternatively, a positive jump may be always followed by a negative jump to capture the rapid decline of electricity prices after a spike. The latter approach is used in the jump diffusion model proposed by Weron et al. [8], which is analyzed in the next Section.

Yet another possibility is to divide the time series into different phases or regimes with different underlying process parameters to be modeled. A jump in electricity prices can then be considered as a change to another regime (the spike regime) that follows a different stochastic process than the so-called 
base regime [20-22]. The switching mechanism is typically assumed to be governed by a random variable that follows a Markov chain with two possible states. Clearly the probability of being (and also staying) in the base regime is supposed to be much higher than that for the spike regime. Regime switching models are also able to consider spikes that last for more than just one day, without the disadvantage of slow mean reversion after a jump.

\section{Empirical analysis}

Having described the stylized facts and the approaches proposed in the literature we are ready to test various spot price models. For the empirical analysis we chose the Nord Pool market daily average system prices (denoted by $P_{t}$ ) for the period January 1, 1997 - April 25, 2000. The choice of the period used in the analysis is not incidental - 1996 was a dry year with exceptionally high electricity prices and the first half of 2000 was used for testing the Asian options pricing model [8,23], since later that year these derivatives were withdrawn from exchange trading.

As stated previously (see the top panel of Fig. 1), the annual cycle can be quite well approximated by a sinusoid of the form $S_{t}=A \sin \left(\frac{2 \pi}{365}(t+B)\right)+C t$, where $A=44.27, B=97.91$ and $C=-0.0271$ are constants (obtained through a least squares fit). Like demand, spot electricity prices are not uniform throughout the week. The intra-week and intra-day variations of demand caused by different level of working activities translate into periodical fluctuations in electricity prices. However, in the present analysis we do not address the issue of intra-day variations and analyze only daily average prices. We deal with the intra-week variations by preprocessing the data using the moving average technique, which reduces to calculating the weekly profile $s_{t}$, i.e. an average week, and subtracting it from the spot prices $[8,24]$. In what follows we model the logarithm of the deseasonalized prices (with respect to the weekly and annual cycles; in short: deseasonalized $\log$-prices) $d_{t}=\log \left(P_{t}-s_{t}-S_{t}\right)$, see the bottom panel of Fig. 1.

\subsection{The jump diffusion model}

Despite their rarity, price spikes are the very motive for designing insurance protection against electricity price movements. This is one of the most serious reasons for including jump components in realistic models of electricity price dynamics. Reflecting the fact that on the daily scale spikes typically do not last more than one time point (i.e. one day), like in [8], we let a positive jump be always followed by a negative jump of about the same magnitude. 
This is achieved by letting the stochastic part $X_{t}$ be independent of the jump component $J_{t} d q_{t}$. For the sake of simplicity we let $J_{t}$ be a lognormal random variable $\log J_{t} \sim N\left(\mu, \rho^{2}\right)$ and $q_{t}$ be a Poisson random variable with intensity $\kappa$. The jump component is estimated from the deseasonalized log-prices $d_{t}$ through a two-step procedure. First, all jumps - defined as price increments exceeding 3 standard deviations of all price changes - are removed from $d_{t}$. Next, the intensity $\kappa$ and the distribution of the magnitude $J_{t}$ of the jumps is estimated from these few selected points (6 in the whole series), yielding $\kappa=0.0050, \mu=-0.8618$ and $\rho=0.5266$.

Putting all the facts together, the jump diffusion model of Weron et al. [8] has the following form:

$$
d_{t}=J_{t} d q_{t}+X_{t} \quad \text { or } \quad P_{t}=s_{t}+S_{t}+e^{J_{t} d q_{t}+X_{t}}
$$

where $X_{t}$ is defined by eqn. (2). The exponent in the last term of eqn. (3) reflects the fact that the marginal distribution of $X_{t}$ is Gaussian, whereas the deseasonalized, with respect to the weekly and annual cycles, and "spikeless" spot prices can be very well described by a lognormal distribution, i.e. their logarithms are approximately Gaussian. The fit is surprisingly good, the BeraJarque test [25] for normality yields a p-value of 0.98 ; for comparison, the pvalue for the "spiky" deseasonalized log-prices $d_{t}$ is less than 0.0001 , allowing us to reject normality at any reasonable level. The parameters of the mean reverting process $(2), \alpha=0.2438, \beta=0.0499$ and $\sigma=0.0529$, were estimated using the Generalized Method of Moments (GMM) $[8,26]$.

\subsection{The regime switching model}

We now turn to regime switching models as, in general, they offer the possibility of having spikes that last for more than just one day. In such models we have an unobservable variable in the time series that switches between a certain number of states and for each state we have an independent price process [26-29]. Additionally we have a probability law that governs the transition from one state to another.

In this Section we construct a two-regime model, although multi-regime models have also been proposed in the literature [21]. It distinguishes between a base mean reverting regime $\left(R_{t}=1\right)$ and a spike regime $\left(R_{t}=2\right)$, i.e. the spot price is supposed to display either mean reverting or jump behavior at each point of time. The price processes $Y_{t, 1}$ and $Y_{t, 2}$ that are linked to each of the two regimes are assumed to be independent of each other. The variable $R_{t}$ that determines the current state is a random variable that follows a Markov chain with two possible states, $R_{t}=\{1,2\}$. The transition matrix $\mathbf{P}$ contains 

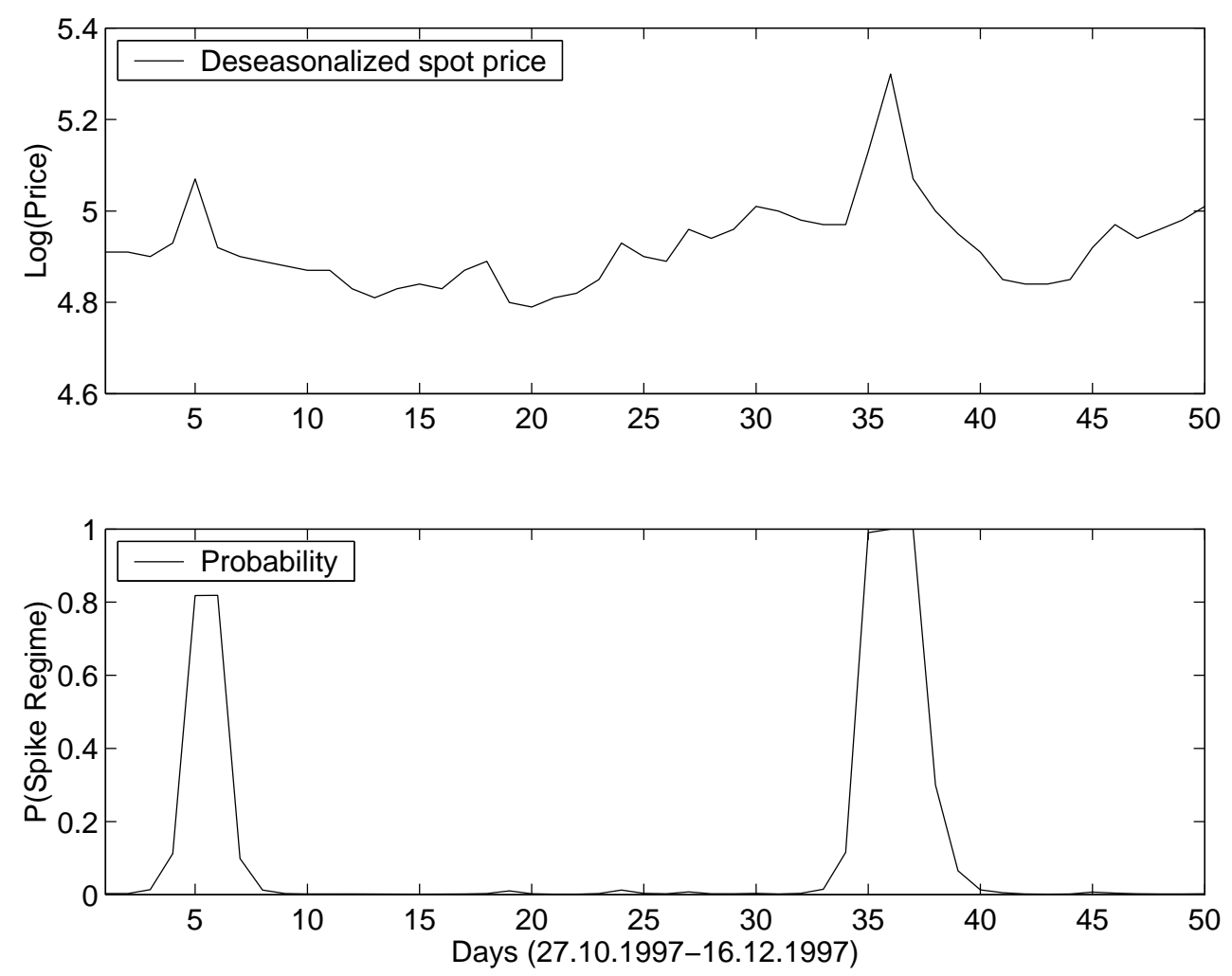

Fig. 2. Top panel: The deseasonalized log-spot price $d_{t}$ since October 27, 1997 until December 16, 1997. Bottom panel: Probability of being in the spike regime for the same time period.

the probabilities $p_{i j}$ of switching from regime $i$ at time $t$ to regime $j$ at time $t+1$ :

$$
\mathbf{P}=\left(p_{i j}\right)=\left(\begin{array}{ll}
p_{11} & p_{12} \\
p_{21} & p_{22}
\end{array}\right)=\left(\begin{array}{cc}
p_{11} & 1-p_{11} \\
1-p_{22} & p_{22}
\end{array}\right)
$$

The current state $R_{t}$ of a Markov chain depends on the past only, through the most recent value $R_{t-1}$ :

$$
P\left\{R_{t}=j \mid R_{t-1}=i, R_{t-2}=k, \ldots\right\}=P\left\{R_{t}=j \mid R_{t-1}=i\right\}=p_{i j}
$$

The probability of being in state $j$ at time $t+m$ starting from state $i$ at time $t$ is given by:

$$
\left(\begin{array}{c}
P\left(R_{t+m}=1 \mid R_{t}=i\right) \\
P\left(R_{t+m}=2 \mid R_{t}=i\right)
\end{array}\right)=\left(\mathbf{P}^{\prime}\right)^{m} \cdot e_{i}
$$

where $\mathbf{P}^{\prime}$ denotes the transpose of $\mathbf{P}$ and $e_{i}$ denotes the $i$ th column of the 
Table 1

Estimation results for the two-regime switching model and the deseasonalized logprice $d_{t}$ for the period January 1, 1997 - April 25, 2000. $E\left(Y_{t, i}\right)$ is the level of mean reversion for the base regime $(i=1)$ or the mean jump size for the spike regime $(i=2), p_{i i}$ is the probability of remaining in the same regime in the next time step, and $P(R=i)$ is the unconditional probability of being in regime $i$.

\begin{tabular}{lcccccc}
\hline & $\beta_{i}$ & $c_{i}$ & $\sigma_{i}^{2}$ & $E\left(Y_{t, i}\right)$ & $p_{i i}$ & $P(R=i)$ \\
\hline Base regime $(i=1)$ & 0.0426 & 0.2078 & 0.0018 & 4.8807 & 0.9800 & 0.9484 \\
Spike regime $(i=2)$ & - & 1.6018 & 0.0024 & 4.9678 & 0.6325 & 0.0516 \\
\hline
\end{tabular}

$2 \times 2$ identity matrix.

Following Huisman and de Jong [22], we let the base regime $\left(R_{t}=1\right)$ be governed by a mean-reverting process, eg. given by the Vasicek SDE (2). However, we let the spike regime $\left(R_{t}=2\right)$ be a lognormal variable, and not a Gaussian one as in [22]. We believe that the spikes (to be more exact: log-spikes or spikes on the log scale) are better approximated by the lognormal than the normal distribution. Hence, the deseasonalized log-price process $d_{t}$ in the two-regime switching model takes the form:

$$
d_{t}= \begin{cases}Y_{t, 1} & \text { in the base regime } \\ Y_{t, 2} & \text { in the spike regime }\end{cases}
$$

where the base regime dynamics are given by $d Y_{t, 1}=\left(c_{1}-\beta_{1} Y_{t, 1}\right) d t+\sigma_{1} d B_{t}$ and $\log \left(Y_{t, 2}\right) \sim \mathrm{N}\left(c_{2}, \sigma_{2}^{2}\right)$.

The parameter estimation can be performed using the EM algorithm [30]. First the whole parameter set is collected in the vector $\theta=\left\{c_{1}, \beta_{1}, \sigma_{1}, c_{2}, \sigma_{2}, p_{11}, p_{22}\right\}$. Then the EM algorithm uses an iterative procedure that basically consists of two parts. In the first step we filter the regime $R_{t}$ at time $t$ of a given data set assuming that we already know the true parameter set $\theta$ of the underlying stochastic processes. We obtain the probability $P\left(R_{t}=j \mid y_{1}, \ldots, y_{T} ; \theta\right)$ that the process was in regime $j$ at time $t$ with knowledge of the complete data set $y_{1}, \ldots, y_{T}$. These probabilities are referred to as smoothed inferences.

In the second step we calculate new maximum likelihood estimates $\hat{\theta}$ for all model parameters. Starting from an arbitrary parameter set $\hat{\theta}^{(0)}$ we calculate new estimates $\hat{\theta}^{(1)}$ using the smoothed inferences from the previous step. With the new vector $\hat{\theta}^{(1)}$ we start the next cycle of the algorithm to reevaluate our smoothed inferences and so on. Every cycle of the EM algorithm produces new estimates $\hat{\theta}^{(n+1)}$ of the unknown parameter set based on the previously calculated value set $\hat{\theta}^{(n)}$. The limit of this sequence of estimates achieves a (local) maximum of the log-likelihood function. 
The estimation results are summarized in Table 1 . The probability of remaining in the same state is very high for the base regime (0.9800) and relatively high (0.6325) for the spike regime, indicating that once we observe a spike another extreme value is quite probable. This is in contrast to the previous jump diffusion model where the price was damped back to the mean reverting level right after the jump.

Note also that for the analyzed time period there is a $5.16 \%$ probability of being in the spike regime. This value is substantially larger than the intensity of the jumps in the jump diffusion model $(\kappa=0.005$ or $0.5 \%)$. However, data points with a high probability of being in the jump regime $\left(P\left\{R_{t}=2\right\}>0.5\right)$ tend to be grouped in blocks. For one jump in the price we get a block of 3-6 such data points, see Fig. 2. If we count the number of blocks it turns out that there are only 20 such periods in the time series, indicating approximately a $1.6 \%$ probability of a spike (or a group of consecutive spikes) occuring at a given time.

\section{Conclusions}

The liberalization of the power markets has created additional risks and new challenges for players in the market. The uniqueness of electricity, discussed in Section 2, distinguishes the power market from other financial or commodity markets and calls for new models of price dynamics. The number of papers addressing these problems is still scarce and the suggested solutions are usually not universal or unsatisfactory.

In this paper we have proposed two distinct, yet in some sense similar models, which recover the main characteristics of electricity spot price dynamics. Both models exhibit seasonality, mean reversion and jump behavior. In both of them seasonality is modeled through a sinusoidal function coupled with a moving average technique for the weekly cycle. Likewise, in both of them mean reversion is achieved through a Vasicek type stochastic differential equation. What distinguishes them is the spike formation mechanism. In the jump diffusion model it is simply an independent homogeneous Poisson process, like the claim arrival point process in risk theory [31], whose parameters are estimated in an ad-hoc manner. In the regime switching model it is the regime switching mechanism itself, which is easily able to produce spikes that last for more than just one day. The estimation scheme is also more objective. This, together with the larger number of the degrees of freedom, in the long term should lead to better estimates and better modeling performance.

Although we believe that both models can be successfully used to price a number of derivatives in the Nordic power market, this claim has to be thor- 
oughly tested. Some preliminary results [23] confirmed the adequacy of the jump diffusion model. Yet, more work still has to be done.

\section{Acknowledgements}

Many thanks to SKM Market Predictor AS for providing the data. All authors would like to acknowledge the support from a joint DAAD-KBN grant (DAAD

008/2002/2003, DZ-KBN 4381/R02/R03). The first author's research was also partially supported by KBN Grant PBZ-KBN 016/P03/99.

\section{References}

[1] D. Pilipovic, Energy Risk: Valuing and Managing Energy Derivatives, McGrawHill, New York, 1998.

[2] V. Kaminski (ed.) Managing Energy Price Risk, Risk Books, London, 1999.

[3] L. Clewlow, C. Strickland, Energy Derivatives - Pricing and Risk Management, Lacima Publications, London, 2000.

[4] A. Eydeland, H. Geman, Fundamentals of Electricity Derivatives in Energy Modeling and the Management of Uncertainty, Risk Books, 2000.

[5] Nord Pool, Nord Pool Annual Report, Nord Pool ASA, 2002.

[6] D.B. Percival, A.T. Walden, Wavelet Methods for Time Series Analysis, Cambridge University Press, Cambridge, 2000.

[7] I. Simonsen, M.H. Jensen, A. Johansen, European Phys. J. B 27 (2002) 583.

[8] R. Weron, I. Simonsen, P. Wilman, Modeling highly volatile and seasonal markets: evidence from the Nord Pool electricity market, in H. Takayasu (ed.), The Application of Econophysics, Springer, Tokyo, 2004, pp. 182-191.

[9] K. Bhanot, J. Risk 2 (2000) 43.

[10] J.J. Lucia, E.S. Schwartz, Rev. Derivatives Research 5 (2002) 5.

[11] E.S. Schwartz, J. Finance 52 (1997) 923.

[12] R. Weron, B. Przybyłowicz, Physica A 283 (2000) 462.

[13] R. Weron, Measuring long-range dependence in electricity prices, in H. Takayasu (ed.), Empirical Science of Financial Fluctuations, Springer, Tokyo, 2002, pp. 110-119.

[14] I. Simonsen, Physica A 322 (2003) 597. 
[15] L. Bachelier, Théorie de la spéculation, Annales Scientifiques de l'Ecole Normale Supérieure III-17 (1900) 21.

[16] P.A. Samuelson, Industrial Management Rev. 6 (1965) 13.

[17] O. Vasicek, J. Financial Economics 5 (1977) 177.

[18] B. Johnson, G. Barz, Selecting stochastic processes for modelling electricity prices, Energy Modeling and the Management of Uncertainty, 1999.

[19] H. Geman, A. Roncoroni, A class of marked point processes for modelling electricity prices, ESSEC Graduate Business School preprint, 2002.

[20] R. Ethier, T. Mount, Estimating the volatility of spot prices in restructured electricity markets and the implications for option values, Cornell University Working Paper 12/1998.

[21] R. Huisman, R. Mahieu, Energy Power Risk Management 5.6 (2001) 32.

[22] R. Huisman, C. de Jong, Energy Power Risk Management 7.11 (2003) 12.

[23] R. Weron, Pricing Asian options in the Nord Pool electricity market, Hugo Steinhaus Center Research Report HSC/03/1, 2003. See:

www.im.pwr.wroc.pl/^hugo/Publications.html.

[24] R. Weron, B., Kozłowska, J., Nowicka-Zagrajek, Physica A 299 (2001) 344.

[25] A. Spanos, Statistical Foundations of Econometric Modelling, Cambridge University Press, 1993.

[26] J.D. Hamilton, Time Series Analysis, Princeton University Press, 1994.

[27] R. Quandt, J. Amer. Statist. Assoc. 55 (1958) 873.

[28] S. Goldfeldt, R. Quandt, J. Econometrics 1 (1973) 3.

[29] J.D. Hamilton, Econometrica 57 (1989) 357.

[30] A. Dempster, N. Laird, D. Rubin, J. Royal Statist. Soc. 39 (1977) 1.

[31] K. Burnecki, W. Härdle, R. Weron, Simulation of risk processes, in J. Teugels, B. Sundt (eds.), Encyclopedia of Actuarial Science, Wiley, Chichester, 2004. 


\section{HSC Research Report Series 2003}

For a complete list please visit http://ideas.repec.org/s/wuu/wpaper.html

01 Modeling electricity prices: Jump diffusion and regime switching by Rafał Weron, Michael Bierbrauer and Stefan Trück 